\title{
La personalità di Pietro Ercole Gherardi nelle lettere al Muratori
}

\section{Antonio Franceschetti}

Settimo in ordine di pubblicazione dei quarantasei annunciati, è uscito recentemente il ventesimo volume dell'Edizione Nazionale del Carteggio di L. A. Muratori (a cura di Guido Pugliese, Firenze, Olschki, 1982, sotto gli auspici del "Centro di Studi Muratoriani di Modena"), comprendente il Carteggio con Pietro E. Gherardi: è composto dalle cinquecentoventidue lettere scritte da quest'ultimo allo studioso modenese in un arco di tempo che va dal 1718 al 1749 e conservate presso la Biblioteca Estense di Modena, con l'eccezione di una, del 15 marzo 1735, che si trova presso l'Archivio di Stato della stessa città. Sarà naturalmente inutile soffermarsi sull'estrema importanza di questa impresa editoriale (inquadrabile nell'ambito del maggiore interesse riservato in questi ultimi anni alla pubblicazione degli epistolari come fonti di rilievo fondamentale per lo studio di un autore e del suo periodo) la quale, in un complesso di oltre ventottomila lettere nella maggior parte inedite, metterà a disposizione degli studiosi un materiale vastissimo, raccogliendolo con criteri piú moderni e con maggiore organicità di quanto l'Epistolario del Muratori edito dal Campori fra il 1901 e il 1922 e le numerose raccolte parziali che ad esso si aggiunsero non abbiano potuto fare, e facilitando l'approfondimento di una serie di aspetti e di situazioni particolari delle quali il critico moderno potrebbe altrimenti rimanere all'oscuro. Basti ricordare, nel caso specifico di questo volume, la lettera del 20 nov. 1745, dalla quale si conosce il sotterfugio dell'editore Pasquali, che, per obbedire agli ordini ricevuti da imprecisate autorità veneziane, nello stampare gli Annali d'Italia mutò in alcune copie pagine originali riguardanti la storia veneta, introducendo delle varianti certamente non attribuibili al Muratori: cosa che, come osservava il Gherardi, "col tempo farà curiosità e darà occasion di lunariare ai posteri" (p. 283).

Se il Muratori si mostrò solerte e assiduo raccoglitore delle lettere dell'amico, conservandone diverse anche quando, per segretezza, per non tradire indiscrezioni e notizie riservate o per altre 
ragioni, questi gliene richiedeva esplicitamente la distruzione dopo la lettura (si vedano ad esempio le lettere del 17 nov. 1723, p. 23 , del 18 nov. 1734 , p. 77 , del 4 apr. 1736, p. 130 , del 20 nov. 1745 , p. 284 , del 26 febb. 1746 , p. 302, ecc.), altrettanto non possiamo purtroppo dire del Gherardi, forse perché la sua vita spesso raminga in conseguenza delle sue mansioni al seguito della famiglia ducale estense non doveva certo facilitare la cosa: come informa il Pugliese nella sua introduzione, nessuna è stato possibile rintracciarne delle, senza alcun dubbio, numerosissime che il $\mathrm{Mu}$ ratori gli indirizzò, e che pure egli dovette in determinate circostanze conservare almeno per qualche tempo (o forse è il caso di pensare che, sopravvissuto all'amico di due anni e mezzo, le distrusse tutte dopo la sua morte?), come ci confermano queste parole: "Io la prego di credere che nelle sue lettere del primo d'ottobre del 1743, de' 13 dicembre del detto anno e de' 24 gennaio del corrente, né pure una sillaba sola a me ella scrisse, a chi dovesse il Monti consegnar le tre copie. . . . Le ho rilette, e di tal cosa ne verbum quidem" ( 8 ag. 1744, p. 214). L'interesse piú immediato di questo volume consiste dunque nella possibilità di ricostruire piú accuratamente la figura e la personalità di questo per altro assai poco noto erudito ed uomo di cultura modenese, del quale il Pugliese dà in maniera succinta (secondo la prassi editoriale stabilita per questo Carteggio dal "Centro di Studi Muratoriani") ma puntuale il profilo nella sua accurata introduzione, ricordandone le opere quasi tutte inedite e delineandone il carattere e in particolar modo i rapporti che egli ebbe con il piú famoso concittadino, al quale rimase legato per tutta la vita da un sentimento di viva ammirazione e di profonda amicizia, e del quale fu sollecito e diligente collaboratore.

Anche se in larga parte gli argomenti trattati nelle sue lettere riguardano notizie e informazioni letterarie di aspetto tecnico, quali acquisti di libri, trascrizioni ed edizioni di opere muratoriane, problemi di rapporti con censori e stampatori e via dicendo, è il caso subito di precisare che la lettura non ne risulta affatto monotona, ma riserva gradevoli sorprese e si rivela spesso attraente e piacevole anche per chi non è direttamente interessato in quegli argomenti. Questo avviene in conseguenza del temperamento del Gherardi, ordinato e meticoloso ma ben lontano dall'essere pedante, il quale si dimostra, come scrive il Pugliese, "osservatore acuto e perspicace che sa cogliere l'essenziale di luoghi e persone, costumi e usanze" (p. 11) e ha dato alle sue lettere un tono spigliato e vivace, introducendovi osservazioni soggettive, giudizi e considerazioni personali su uomini e cose con i quali venne a con- 
tatto nel corso della sua vita e sui quali non esitò a pronunciarsi apertamente molto spesso con l'amico. Veniamo cosí a conoscere ad esempio la sua favorevole opinione su Apostolo Zeno, da lui frequentato regolarmente sia durante un soggiorno a Vienna, sia nel lungo periodo di tempo trascorso a Venezia, del quale ricorda spesso i sentimenti di buona amicizia per il Muratori (lettere del 19 nov. e dell'11 dic. 1723 , pp. 23 e 29, del 5 e del 26 genn. 1724, pp. 32 e 37, ecc.) e il lusinghiero giudizio sugli Annali d'Italia, opera "dotta, maestrevole, sugosa, franca, prudente e veritiera" (27 febb. 1745, p. 240) da lui letta con "ammirazione e piacere" e lodata "in privato e in pubblico" (29 mag. 1745, p. 256), mentre il Gherardi a sua volta ne ammira la "bella raccolta d'ogni sorta di libri stampati, di rare edizioni, e d'ottimi autori" e l'altra "altrettanto bella . . . de' manuscritti, in gran parte de' secoli bassi" (24 nov. 1742, p. 149; raccolte che in buona parte si trovano ora presso la Biblioteca Marciana di Venezia), o le Annotazioni alla Biblioteca dell'Eloquenza Italiana del Fontanini, "dotto e sugoso libro di censura modesta e ben fondata" (27 nov. 1745, p. 284), che l'autore tuttavia preferí non pubblicare in vita e che avrebbe visto la luce solo dopo la sua morte, a cura dell'allievo Marco Forcellini (Venezia, Pasquali, 1753, voll. 2). (Degli incontri veneziani ci ha lasciato ricordo anche lo Zeno nelle sue lettere al Muratori, pubblicate sempre in questo Carteggio, vol. 46, a cura di Anna Burlini Calapaj, 1975, pp. 191-409). Interessante per lumeggiare piú puntualmente il carattere del Gherardi è quanto leggiamo nell'ultima, del 16 febb. 1743: "Non passa settimana ch'io non sia con l'amico Girardi o ch'egli non sia con me. Ogni volta si parla di voi, e la vostra degna persona è ' 1 piú dolce condimento della nostra allegra conversazione. Non so qual ritratto egli vi abbia fatto di me: forse non molto vantaggioso: comunque ne sia, mi è forza vendicarmene col rappresentarvelo un gran traccanatore del miglior vino ch'io m'abbia, a segno che di mezzo a qualche suo racconto perde la memoria, quasi bevuto avesse di Lete: onde va in collera contro se stesso, si rammarica e sino s'infuria e ne freme. Proccuro di consolarlo e di rimetterlo in quiete, ma nulla giova; e pieno del suo rancore se ne va frettoloso e mi lascia. Voi che ne dite? e a me ne date la colpa, io la ributto in lui, ed egli si scarica su quel vino di Corfú di cui è troppo gourmand ed intemperante. Esortatelo ad esser piú sobrio, e la memoria tornerà a suo segno," p. 409. Il Muratori dovette in effetti scriverne al Gherardi, che rigetta per altro l'accusa nella lettera del 2 mar. 1743 con queste parole: "Carità pelosa è quella dell'amicissimo nostro sig. Apostolo. Spigne egli gli amici suoi con gl'inviti e lusinghe a scostarsi alcun 
poco dalla temperanza. Dapoiché ha il piacere di vederne esilarati gli spiriti dall'apprestato nettare greco; accagiona dipoi i meschinelli se non se di dichiarata ubbriachezza, almeno di allegria alcun poco straordinaria. Giove e Bacco gliela perdonino! Buon per me che, messo nell'occasion prossima del piacere, ho nulladimeno tanto di ritegno da non annegarmivi dentro. Non avrà, no, il compassionevole cuor muratoriano o il dispiacere o 'l contento di vedermi, sovrafatto dalle bevande di vin di Cipro, andare a nascondere in una di queste puzzolenti cloache..." p. 160).

Ancor piú profonda fu la stima del Gherardi per un altro amico veneto del Muratori, Antonio Conti, da lui giudicato "l'unico dotto vero in tutta Venezia, e fuori anche per molte miglia" (6 genn. 1748 , p. 411), e del quale apprezza sia le opere che il metodo di lavoro (lettere del 29 mar. e del 5 lug. 1743, pp. 165 e $180-181$, del 29 genn. 1746, p. 298, ecc.), suggerendo all'amico in una circostanza di scrivergli direttamente "per trarne consiglio e lume" (19 lug. 1743, p. 181). D'altro canto egli non esita invece a censurare i letterati italiani incontrati a Vienna con queste parole: "della gloria di nostra nazione poco anzi nulla si curano. Amano e cercano essi il particolare loro vantaggio, non già il bene, il decoro e 'l buon nome universale. Non credo che si possa in altro luogo giammai rinvenir gente e persone tanto tra di loro divise quanto questi nostri" (17 nov. 1723, p. 21); né alcuna simpatia ebbe mai per Scipione Maffei, da lui regolarmente trattato con ironia o quanto meno con indifferenza, e molto spesso criticato e denigrato aspramente, come quando sottolinea le sue deficienze come teologo (lettere del 2 genn. 1743, p. 155 e del 22 ott. 1746, p. 333), e gioisce per il modo in cui il Voltaire, a proposito della Merope, l'abbia "con civiltà, cerimonie e lodi stafilato non poco" (22 ag. 1744 , p. 215), e del quale ci lascia in una delle sue ultime lettere questo feroce giudizio: "Il lupo perde il pelo, ma non mai il vizio. Amicizia non ha per alcuno il marchese Maffei. La vanità e la presunzione sono e saranno finché vivrà le passioni predominanti, che gli faranno dimenticare i doveri di civiltà e di riguardo verso di qualunque persona. . . . Minchione ch'egli è!" (3 magg. 1749, p. 496).

Il Gherardi ci si presenta dunque, possiamo subito osservare, come un individuo con dei limiti ben precisi da un punto di vista concreto, con simpatie e antipatie ben definite anche se non sempre valide storicamente, con mentalità, gusti, idee che possono essere considerati, e lo sono spesso in realtà, quelli di una persona media - il che certo non implica necessariamente mediocre - non dotata di qualità superiori intellettualmente o cultu- 
ralmente a quelle del suo tempo e del suo mondo: ma la sua personalità 'media' finisce con il coinvolgere il lettore delle sue lettere proprio per quel carattere di schiettezza, di sincerità e di semplicità che le condiziona, per il ritratto che ne risulta di un 'settecentista' assai lontano da quelli tutti letterari del fragile Metastasio da un lato contrapposto all'altro del virilissimo Alfieri, del superficiale Algarotti, del sorridente Goldoni e via dicendo: il ritratto di un personaggio che non ci si sente necessariamente in dovere di ammirare o di stigmatizzare, ma al quale ci si può avvicinare accettandolo per quello che $\mathrm{fu}$, vale a dire, come lui stesso felicemente si autodefinisce, un uomo dal "naturale alquanto lieto e disimbarazzato" (21 giu. 1743, p. 179), che non condivide con i suoi contemporanei la passione per i teatri e per i divertimenti ma si accontenta delle sue abitudini semplici, tenendosi lontano dal "desinare o cenare a casa d'altri . . dal gustare acquevite, rosoli, né vini forestieri . . . da bevande di ciocolata, caffè e sorbetti" ed evitando "collezioni, merende, smangiucchi fuori di pasto" (25 febb. 1747, p. 358); pronto a inalberarsi con l'amico se gli sembra che questi lo accusi di non aver tenuto abbastanza bene la loro contabilità ("mi creda un po' piú giusto e meno interessato" gli scrive il 18 mar. 1736, p. 129), ma pronto anche a perderci del suo pur di assicurargli l'acquisto di libri importanti per il suo lavoro (17 ag. 1743, p. 182); che avrebbe ben volentieri trascorso la vita nella sua Modena fra libri e carte e considerò "uno sconcerto della vetusta sua professione" quello che tanti altri avrebbero reputato "fortuna e grazia," l'esser chiamato al ruolo di "precettore abicidario" dei "serenissimi principini" estensi ("Oh va' mo ad istudiar per tutto il tempo di vita tua cose erudite e sublimi per aver poscia tutto ad un tratto a ridurti ad insegnare altrui l'alfabeto!" 7 ott. 1734, pp. 71-72), attività che lo costrinse a molti anni di peregrinazioni al seguito della corte, che non amò mai e della quale non condivise per nulla i piaceri (non sempre molti in ogni modo, a giudicare da come egli descrive ad esempio una villeggiatura a Sassuolo: "Questo nostro soggiorno è piú da persone di vita eremitica che da gente di corte principesca. Tedio, stufaggine e melanconia in moltissimi, e scontentezza in tutti. Quel poco di filosofia che ho appreso in passato, unita al mio natural piú tosto allegro, mi van tenendo forte, ond'io non m'abbandoni al comune languore e abbattimento di spirito che in faccia ad ognuno qui chiaro si vede," 9 sett. 1738, p. 140).

Del Gherardi non si può certo dire che abbia avuto la passione del viaggiare o spartito con tanti uomini del suo secolo alcuna curiosità e bramosia istintiva di conoscere nuovi popoli e di visitare 
città e paesi lontani; la sua anima cosí semplicemente e spontaneamente domestica risalta in maniera chiarissima nelle numerose pagine in cui egli si lamenta per la lontananza dalla città natale e per la costrizione di vivere in luoghi che non apprezza, in mezzo a popolazioni di cui egli vede sempre il lato negativo e per le quali non nutre né comprensione né interesse. Cosí Vienna gli sembra una "galera, dove l'avarizia, le risse, le competenze, le invidie, con tutti gli altri peccati mortali annidano" (23 febb. 1724, p. 43) e dove regnano non "Pallade, ma bensí e Venere e Mercurio e Bacco e quant'altri iniquissimi falsi numi seppe inventarsi il ceco gentilesimo" (12 apr. 1724, p. 51); non ne sopporta il clima che lo costringe, se desidera "andar fuor di casa pe' tempi nevosi, piovosi, fangosissimi, a portar di dí e di notte gli stivali" (5 apr. 1724, p. 50) e che "non ha metodo di tempi, e dove ogni stagione è confusa senza cominciamento e senza termine, e dove non avvi giornata in cui ognora non si faccia cangiamento di cielo," causandogli delle complicatissime malattie "di respiro, di tosse, di vigilie, d'inappetenza, di fiacchezza e d'altri sintomi . . . assai e poi assai molestissimi" (10 mag. 1724, p. 54); i Tedeschi sono per lui "orsi . . . e nel tratto e nel pensiero e nel vitto e nel modo di vivere" (24 nov. 1723, p. 25), hanno come "primi principi . . . l'ignoranza, la crapola, il far bezzi" (1 dic. 1723, p. 28), né esita a deridere il fatto che nella loro lingua, "al contrario delle altre nazioni e del sentimento universale," sono "di genere maschile la luna, di femminile il sole, di comune la donna" (ibidem), o la loro abitudine di tenere "basette o ... mustacchi ... sí lunghi e folti che intrecciandoli se li legano ad ambe le orecchie" (26 genn. 1724, pp. 37-38).

Ma non si pensi che questa insofferenza e questa, si direbbe, istintiva ostilità verso la capitale di Carlo VI, il suo clima e i suoi abitanti siano attribuibili esclusivamente a un sentimento di antipatia per un popolo straniero: il concittadino del Tassoni non si mostra certo piú tenero o piú comprensivo con i vicini Bolognesi, da lui spesso ironicamente chiamati "Petroni" o "Felsinei," e che egli giudica "nati fatti per la sordidezza dell'interesse e nemici d'ogni onesto forestiere, non che delle sante leggi di una sincera civil società e commercio" (9 mar. 1735, p. 81), "becchi foderati" (6 ott. 1735 , p. 102), "mendaci" (15 ott. 1735, p. 104), "appassionati sciocchi" e "pecoroni" (23 nov. 1735, p. 108); ed anche a Bologna si lamenta per le "flussioni, dalle quali nel verno . . . incostantissimo, e d'umido e freddo essai ineguale, è toccato su non poco negli articoli delle mani," giungendo a dire di esserne cosí "stucco, stuffo, annoiato" da desiderare quasi di ritrovarsi piuttosto a Vienna (29 genn. 1736, pp. 123-124). Né molto diversi i 
suoi sentimenti e i suoi giudizi sulla città dove trascorse piú di sette anni in quanto vi si era trasferita la famiglia ducale durante la guerra di successione austriaca: la "scandalosa Venezia ... nella quale . . . si mena la vita di Petronio Arbitro" (28 sett. 1747, p. 394). Se da un lato egli apprezza in quella circostanza la politica del "saggio veneto governo" per la sua "neutralità," per la sua "buona guardia" del territorio e per le disposizioni a tutela della "pubblica sanità" (11 magg. 1743, p. 173), e in altra occasione la sua "prudenza e precauzion" a proposito di un'epidemia del bestiame scoppiata nel territorio di Dolo, superiori (si noti bene) forse anche a quelle che avrebbero in analoga circostanza applicato i "magistrati di Modena e di Reggio" (7 ott. 1747, p. 397), dall'altro cosí ne sintetizza negativamente la politica interna: "Spettacoli, teatri e divertimenti pubblici per la maggior parte dell'anno; funzioni solenni di santi, feste di chiese, e concorso di gente alle divozioni nell'altra parte del minore. Divertiti cosí ed occupati i sudditi non pensano che a passar dai solazzi alla divozione, e da questa a quelli. Cosí si vive con sicurezza e con quiete" (22 apr. 1747, p. 368). E come conseguenza "costí ognuno in mezzo alle angustie, alle miserie si vuole spassare e divertire . . . cotesta popolazione tutta tutta va in malora cantando. Debiti a iosa, spiantamenti di case e di famiglie senza riparo, ed altri disordini" (24 febb. 1748, pp. 414-415); le passioni predominanti sono quelle "della regatta . . . del giuoco e della femmina" (29 sett. 1744, p. 222), la città "è il vero paese de' pazzi e la reggia della sensualità e dell'avarizia" (12 nov. 1746, p. 337) e quando finalmente si profila vicino il ritorno in patria, il Gherardi scrivendo all'amico lontano trova solo queste parole per congedarsi dalla città dove tanti anni aveva vissuto: "Dio ci liberi da questo pestifero lungo soggiorno, che è stato ed è la rovina di tutto quel bene che la religione e la prudenza degli antenati nostri aveano stabilito. Fra i non pochi gastighi di Dio, questo di venire a Venezia è stato il maggiore" (29 giu. 1748, p. 438).

Parlare a questo punto di un'incontenibile xenofobia da parte del Gherardi può essere in linea di massima giustificato: tuttavia, per capire meglio il suo atteggiamento (non certo per accettarlo o scusarlo), sarà il caso di tener presente non solo il suo spirito tranquillo, il suo castigato senso morale e "l'amor che naturalmente ognuno porta al proprio nido" (20 genn. 1736, p. 123), ma anche il suo vivo senso di partecipazione per le sventure "dell'afflitta e scarnificata . . . Italia" (18 nov. 1747, p. 406) in quel nefasto primo mezzo secolo del Settecento che vide un susseguirsi di occupazioni, di invasioni, di angherie e di soprusi da parte di eserciti 
stranieri in varie zone della penisola, incluso, certo non fra gli ultimi, il suo amatissimo ducato di Modena e Reggio - e da questo punto di vista anche il "Re Sardo" diventa un tiranno scorticatore di "cotesto miserabil cadavere di paese," nelle lettere del 19 ott. e del 21 dic. 1748 (pp. 468-469 e 476-477). Si spiegano cosí i suoi sfoghi contro "il fanatismo inglese, l'alterigia spagnuola, la superbia tedesca e l'ambizion franzese" (8 apr. 1747, p. 366), contro "le nazioni oltramontane, egualmente tutte nemiche detestabili della . . . misera Italia" (13 magg. 1747, p. 372), tutte congiurate a derubarla e ad assassinarla, e le sue invettive contro quegli Italiani "ossessi dallo strabocchevole genio" per gli stranieri (ibidem) che non condividono la sua xenofobia: "Sia pur frustato dal boia, chi possa giammai per verun oltremontan fomentare veruna inclinazione o genialità! Ma il dolerci non giova. Ragghiare e portar la soma e aver le bastonate" (14 nov. 1735, p. 106).

Questo spirito nazionalistico che gli suggerisce spesso espressioni di amaro compianto per le condizioni del suo ducato e dell'Italia tutta si traduce anche naturalmente in aperta commiserazione per le sventurate popolazioni, per le classi piú povere che sono piú facilmente e piú immediatamente vittime della situazione. Cosí egli critica i Bolognesi che "per non volersi scomodare ... dando il quartier di dentro alla città a' Tedeschi, vogliono acquartierarli nel contado, nelle castellanze e nel territorio, per mandare in esterminio tutta la campagna" (4 dic. 1735, p. 113); durante la guerra di successione austriaca, a proposito di uno scontro fra eserciti nemici, lamenta che esso sia stato "la rovina delle ville da basso nel Modenese col saccheggio permesso e fatto dare alle medesime dal generale Traun" (22 mar. 1743, p. 164), accusa il "danno grande e peggiore sconcerto" che ne deriva al commercio (8 ag. 1744, p. 214), si rammarica per il "milione e ducentomila ducati" spesi dalla famiglia ducale durante il soggiorno a Venezia, che sarebbero stati tanto utili a "cotesto spiantato e miserabile ... paese" (9 ag. 1749, p. 514); paese che egli, per colpa della "guerra desolatrice di beni e sustanze," non si aspetta di ritrovare al suo ritorno "se non se uno scheletro d'ossa spolpate" (24 febb. 1748, p. 415). E nella stessa direzione non mancano nelle sue lettere esplicite condanne per il comportamento dei potenti e dei ricchi, indifferenti alle sofferenze dei poveri e alle conseguenze che il loro sconsiderato modo di agire arreca a questi ultimi. Non ci sono dubbi nella sua mente sulla lealtà e sulla fedeltà da lui dovuta al Duca di Modena e Reggio, sulla bontà del sistema monarchico e sulla necessità per tutti di osservare una "ubbidienza legittima" al "naturale . . . Sovrano" (22 mar. 1743, p. 164); 
egli non esita a condannare la Massoneria in quanto la vede ispirarsi alla "iniqua massima" di "quel furbo di Cromuele . . . autor della morte data al re Carlo Stuardo," il quale "avrebbe voluto introdurre in Inghilterra non meno che negli altri reami il sistema repubblichistico e la depression de' monarchi" sostenendo come principi assoluti "la libertà e l'uguaglianza" (15 lug. 1747, p. 383), e afferma esplicitamente: "La mia curiosità nella lettura dei due libercoli de' Franchi Muratori, se non $\mathrm{m}^{\prime}$ ha fatto imparar cosa alcuna d'importanza, ha certo fatto almeno a me conoscere le ridicolosità e pazzie delle teste degli uomini, e mi ha pienamente informato dell'estrinseco d'una società sterminata di gente che colle inezie loro ha perfino allarmato le corti $\mathrm{d}^{\prime}$ Europa . . . i io non ne ho formato se non il concetto di una massa di matti" (29 lug. 1747, p. 385). Ma questo non gli impedisce di ironizzare sul pellegrinaggio a Loreto organizzato dalle figlie di Rinaldo I, per il quale "si spenderanno molte migliaia di zecchini e d'ungheri" (4 apr. 1736, p. 130); di deridere il fatto che lo stesso Duca, la cui "attentione ... è troppo occupata da rilevanti circostanze che piú gl'importano," non si occupi delle suppliche "che chiedono spedizione e non l'ottengono," dato che "di tal peso, di tal forza sono appunto le poco gustose contingenze presenti che, penetrando al vivo lo spirito di chi governa, lo sconvolgono e il pongono in sí fatto accendimento bilioso e irascibile, onde poi ne succedono nel temperamento sincopi e deliqui, a' quali una pronta apritura di vena ripara e ricompone nel suo equilibrio gli umori e 'l fluido che di soverchio bolliva" (7 apr. 1736, p. 131); di osservare che "i principi d'oggidí sono assai deboli . . . effetto di troppa stima di se medesimi" (19 apr. 1743, p. 169); di censurare la nobiltà modenese che in una circostanza a Venezia, indifferente alle sciagure e alla miseria conseguenti alla guerra, ha "progettato e stabilito di adunarsi a ballare e a buttar via danaro" (24 febb. 1748, p. 415); di commentare che "la pace fatta è una vera ladreria e un manifesto assassinio" per le "nuove contribuzioni" che minacciano gli abitanti del ducato (7 dic. 1748, p. 475); di stigmatizzare lo spreco del denaro, col quale "si sarebbe potuto aiutar tanti poveri," speso da Isabella Foscarini Corner per dare una festa d'addio alla famiglia ducale prima della partenza da Venezia (26 lug. 1749, p. 510).

Questo stesso atteggiamento di comprensione e di benevola partecipazione per i problemi delle classi meno abbienti - che lo indusse una volta a intervenire direttamente presso il Muratori allo scopo di aiutare "in tutta confidenza e segretezza" due stampatori del Pasquali, che rischiavano il licenziamento per aver smarrito alcune pagine con l'originale di una sezione degli Annali 
d'Italia (15 febb. 1744, p. 195) - condiziona la sua posizione nettamente favorevole alla riduzione del numero delle festività religiose (un punto assai dibattuto nel corso del Settecento, e che coinvolgeva direttamente proprio quelle classi, in quanto in tali giornate non era consentito lavorare) e la sua aperta disistima ed antipatia per quegli esponenti del mondo clericale che ne approfittavano per smungere il piú possibile non solo i ricchi, ma anche i poveri. Il Gherardi fu senza dubbio un'anima profondamente e intimamente credente: numerosissime sono nelle sue lettere le frasi in cui si parla della guerra come un castigo inviato da Dio, e si sostiene la necessità di pentirsi dei propri peccati, di umiliarsi a Lui e di accettare i decreti della Sua volontà. Ma questo sentimento religioso della vita non gli impedisce di riconoscere e di condannare il comportamento egoistico ed interessato del clero ove gli sembri necessario. Cosí egli osserva ad esempio: "gli operai e gli artisti moderni, a cagion delle troppe feste, lavoran molto poco fra l'anno e, dalla frequente occasione di non far nulla, s'impoltroniscono e vanno abiurando i mestieri. S'aggiunga che per gli operai ogni dí di festa s'ha da contare per due successive giornate, l'una festiva in cui si perdono nelle osterie a giocare e ad ubbriacarsi, e l'altra seguente in cui smaltiscono le non peranche digerite ubbriachezze. A conto giusto non lavorano un terzo in tutto l'anno" (22 dic. 1742, p. 153); e ancora: "continuano i preti e i frati a vindemiare alle spalle di questa ignorantissima gente. Poco importa se l'arti e i mestieri s'imbastardiscono, se si moltiplica a dismisura la folla degli oziosi, de' ladri, de' poveri, se crescono alle taverne e ai postriboli meretrici ed ubbriaconi e solennissime birbe. Una sola val per tutte. Frate domenicano non c'è che, facendo i dí di festa recitare o nella mattina o verso la sera in chiesa il rosario, non profitti ogni anno a forza di ridicoli esempli, di fastidiose esortazioni e di minaccie di dannazion eterna, trecento e quattrocento ducati di sua porzione, altrettanta restandone al pievano e parrocco, nella cui chiesa va il rosariante smugnendo le borse a questo popolo semplicione. E questa novità si lascia correre da un governo capital nimico d'ogni novità, solo perché essa contribuisce a svagare e divertire la gente col mantello della divozione" (15 febb. 1749 , p. 485). Irrita particolarmente il Gherardi il modo in cui a Venezia si sfruttano l'ingenuità e la buona fede popolari per "far buona raccolta, sotto titolo di carità e di limosina, di contante," mandando in giro "quasi ogni giorno per le contrade i destinati bussolanti che, ad alta voce nominando i santi favoriti, aspettano che i divoti buttino loro giú dai balconi soldi e soldoni"; oppure si vedono "or qua or là per le strade vestiti del sacco, se- 
condo la divisa delle scuole, uno scritturale con tre facchini portanti un enorme libraccio, su cui stan notati i nomi e le case d'uomini e donne ascritte alla compagnia di quel tal santo e tassati a pagare un tanto, quali esattori divoti riscuotere nanti la porta della casa dell'associato la tassa impostagli" (26 febb. 1746, p. 301). Né molto diverso o piú intelligente è il comportamento dei ricchi che credono "di acquistare il paradiso col cacciare in corpo a un centinaio di frati, in una settimana, ciò che distribuito con carità prudente potrebbe per piú d'un mese servir d'alimento a piú centinaia di poverelli" (6 lug. 1748, p. 440). E chi sono poi questi sfruttatori della sciocca semplicità dei credenti? "Se darete . . . un' occhiata al clero secolare, zoppi, storpi, gobbi e assiderati vi si presenteranno per sacerdoti e ministri del sacro altare in qualunque parrocchia, gente tutta di schiatta di barcaiuoli e facchini, senza creanza, senza lettere, senza costume" (15 febb. 1749, p. 485), la cui ignoranza è evidente non appena aprono bocca in una chiesa: "qui avviene alla predicazione della divina parola ció che succede alla profession della musica. Il troppo numero de' cantanti, che per saper quattro note s'espongono subito al pubblico, non è dissomiglievol da quello de' sacri oratori, che con quattro passi di Scrittura e con pochi testi storpiati di santi Padri spacciano sui pulpiti la corta loro mercatanzia" (8 mar. 1743, p. 162). Ad essi il Gherardi non può che contrapporre con ammirazione $\mathrm{i}$ monaci armeni del convento dell'isola di S. Lazzaro, "persone di condizion nobile o almeno riputata molto civile da loro . . . tutti di un tratto dolce, rispettoso, di soda umiltà, e di sí buon contegno che insegnar potrebbe a tanti e tanti nostri Italiani, palloni pieni di vento, la cortesia, e il modo di rispettare e d'essere rispettato . . . potrebbono a tutti servir di modello di civiltà disinvolta" (3 giu. 1743 , p. 176); per quanto poi, a proposito della traduzione latina di un loro manoscritto di S. Iacopo di Nisibia che il Muratori desiderava ottenere, egli non possa trattenersi dall'esclamare anche nei loro riguardi: "non sa ella che qui non si muove un dito che a spinta di danaro? E monaci e frati e preti e secolari non pensano ad altro che all'interesse. Nei paesi grandi il guadagno è il primario e potente loro avvocato non già presso Dio ma presso il diavolo. Se ella non è in istato di spendere, dimetta il pensiero di avere tradotto il Nisibeno" (31 ag. 1743, p. 184).

$\mathrm{Ma}$ il ritratto che risulta del Gherardi dai suoi vari atteggiamenti quali siamo venuti delineando nel corso di queste pagine non sarebbe completo se, alle note umane e realistiche, non si aggiungesse il suono della corda che piú spesso di ogni altra è toccata dal corrispondente di questo carteggio, la nota che suona forse piú 
gradita allo studioso del Muratori e al lettore in generale: quella della sua amicizia umile e disinteressata per il piú grande concittadino (del quale non c'è dubbio che egli si sia considerato in certo modo aiutante e assistente a un livello inferiore), della sua costante preoccupazione per il successo delle sue opere e per il riconoscimento dei suoi meriti, della sua, piú intima e affettuosa, cura per il suo benessere e per la sua salute fisica. In questa dimensione scopriamo un Gherardi che, come scrive benissimo il $\mathrm{Pu}$ gliese, "si comportava da amico fedele e da esortatore perché candidamente persuaso della grandezza del Vignolese" (p. 8); un Gherardi che in una delle ultime lettere da Venezia - precedente solo di pochi mesi la morte dell'amico - poteva scrivergli: "Dio, che mi vede nel cuore, sa ch'io vorrei poter perpetuare la degna persona sua. Secoli passeranno che Modena né l'Italia non vedrà un Muratori," aggiungendo subito dopo con modestia nei propri confronti, evidentemente in risposta ad elogi da lui ricevuti: "Quanto piú vo avanzandomi nell'età, tanto piú vo conoscendo la necessità di studiare l'interno mio libro, per riconoscere me stesso. Povero ed ignorante sempre piú mi ravviso, ed ella ha un concetto di me ch'io so di non meritarlo" (17 lug. 1749, p. 508).

Lo vediamo cosí occasionalmente proporre cambiamenti all'amico, come a proposito del titolo dell'opera Il Cristianesimo felice nelle Missioni dei Padri della Compagnia di Gesú nel Paraguay, in cui l'aggettivo sostituisce un originario "felicissimo" secondo il suo consiglio (cfr. le lettere del 15 e del 28 dic. 1742, pp. 151-152 e 153) - si tratta di consigli che il Muratori non accettava automaticamente, come nel caso di quello Dei difetti della giurisprudenza, che non diventò "Dei difetti dell'uso o nella pratica della giurisprudenza" (26 apr. 1743, p. 170) - raccomandargli editori per esempio Girolamo Albrizzi, da lui moltissime volte sostenuto a cominciare dalla lettera del 31 ag. 1743, p. 184, fino a quando non lo deluse, a proposito della pubblicazione del trattato muratoriano Della pubblica felicità, oggetto de' buoni Principi, per la sua "sciocchezza" e "balordaggine," 26 lug. 1749, p. 510), o suggerirgli aggiunte e modifiche per le sue opere (28 nov. 1744, pp. 227-228). Ma lo vediamo soprattutto preoccuparsi per l'eccesso di lavoro cui si sottoponeva il Muratori (sono ben noti i due versi da lui scritti ancora ventenne: "Non la quiete ma il mutar fatica / alla fatica sia solo ristoro") e per le sue condizioni di salute; fin da una delle prime lettere che ci sono rimaste leggiamo queste parole: "risovvengasi dell'anno passato, alloracché stette sí male. Abbia dunque e metodo e sobrietà nell'applicare. Mi si scrive da chi la va osservando che se le conosce nel sembiante lo troppo studiare e 
faticar con la testa" (1 ag. 1721, p. 16). Raccomandazioni simili costituiscono un vero luogo comune nel corso di questo carteggio: per quanto il Gherardi si dica convinto che "la passione dello studiare è diventata nello spirito e nel temperamento muratoriano natura, e non c'è piú caso di privarsene né di abbandonarla" (21 apr. 1732, p. 69) e che l'amico "morrà a suo tempo co' libri in mano" (12 giu. 1743, p. 178), egli non cessa di raccomandargli "ilarità d'animo, buona dieta e riposo" (1 ott. 1746, p. 328), "riguardo e poi riguardo" (15 ott. 1746, p. 331), di "porre . . ogni attenzione e premura per riacquistare nell'ore notturne una moderata continuazion di riposo e di sonno, necessarissimo alle concozioni e digestion dello stomaco" (28 sett. 1747, p. 393), di "moderarsi, svagarsi, villeggiare, quietarsi, sí per cercar di rimettere gli spiriti perduti, come per conservarne il resto" (15 giu. 1748, p. 434), di rinunciare a "cioccolatte, caffè, tabacco e licori" come aveva fatto il padre del Gherardi stesso, il quale "campò centotré anni" e "nella sua professione faticò moltissimo, si mosse assai e visse sano" (17 lug. 1749, p. 508). Toccando poi un argomento che pensava dovesse certo far presa sull'animo del religiosissimo $\mathrm{Mu}$ ratori, quando questi ebbe compiuto i settantacinque anni, il Gherardi cosí lo ammonisce: "Abbia . . . un po' piú di carità a se medesima e, da che la Provvidenza le ha donato un vigoroso temperamento, non l'infievolisca colla troppa applicazione, acciocché possa vivendo continuare a servir Dio e a giovare co' scritti suoi al prossimo" (29 ott. 1746, p. 334); e in altra circostanza gli ricorda che "della sanità corporea dataci da Dio noi dobbiam dargli conto, godendola in usufrutto e non già in proprietà" (2 sett. 1747, p. 392). Infine in una lettera che sembra quasi un addio e che precedette solo di pochi mesi la morte del Muratori, dopo avergli data notizia di quella del libraio veneziano Lorenzo Basegio e di una paralisi che aveva colto, fortunatemente solo per breve tempo, Apostolo Zeno, egli cosí prosegue: "Amato mio Muratori, abbiamo un po' piú di carità per noi stessi. Se per vivere sano, occorresse lasciar stare nelle scanzie i libri, vi si lascino. Oro né gloria non ha il mondo che possa pagare la salute di mente e di corpo. Ella ha già fatto il suo credito fra gli uomini, ha fatto di gran bene al pubblico, ha mostrato come si dovrebbe pensare e comporre, ha rischiarato tante tenebre d'ignoranza che restavano, ha mossi e spronati tanti e tanti ad applicarsi a studi utili e sodi. Tempo è oramai di riposare" (28 giu. 1749, p. 505). Queste parole, uscite dalla penna di un uomo che aveva altre volte, come osserva giustamente il Pugliese, spronato l'amico "ad ulteriori fatiche" (p. 8), soprattutto nei momenti in cui questi non gli mandava nulla da 
fare, sono senza dubbio particolarmente significative e commoventi.

In conclusione possiamo affermare che la lettura di questo carteggio permette certo di conoscere molto piú intimamente e profondamente una figura che merita, ci sembra, maggiore attenzione di quella, scarsissima, che gli è stata finora riservata - e basti vedere in proposito l'esiguità quasi incredibile dei riferimenti bibliografici contenuta nell'introduzione del Pugliese. C'è da augurarsi che la pubblicazione di questo volume induca a riprendere in mano anche le altre sue opere, che possono forse riservarci altre piacevoli sorprese su questo personaggio, ed aiutare a definire piú precisamente la sua presenza e il suo rilievo nell'arco del Settecento italiano.

University of Toronto. 